\title{
Asociación entre el nivel de actividad física y la percepción de la calidad de vida en personal administrativo durante la pandemia del covid-19 en una universidad privada del Perú.
}

Association between the level of physical activity and the perception of the quality of life in administrative personnel during the covid-19 pandemic in a private university in Peru.

Aracelly Stefany Yanamango Castillo ${ }^{1}$, Crhistian Jony Ulirido Horna Díaz ${ }^{1}$, Vilma Violeta Lizana Villegas ${ }^{1}$, Liz Angélica Ramos Quenallata ${ }^{1}$, Ana María Huambachano Coll Cárdenas ${ }^{2}$, Gino Mauricio Calvo Moreno ${ }^{1}$

\section{RESUMEN}

La actividad física y la percepción de calidad de vida tienen importancia para conocer los problemas del sedentarismo, enfermedades no transmisibles e inactividad física en la población. El COVID-19 es causante de la emergencia sanitaria según la Organización Mundial de la Salud (OMS). En Perú, el estado de emergencia sanitaria establecido obligó a enfrentar desafíos laborales, por ende, inició el teletrabajo como alternativa laboral, la cual asocia a una vida sedentaria sin actividad física y dolores crónicos al aspecto biopsicosocial. Objetivo: Determinar la asociación entre el nivel de actividad física y la percepción de la calidad de vida en el personal administrativo durante la pandemia del COVID-19 en una Universidad Privada del Perú. Material y métodos: Estudio de naturaleza observacional, analítico de corte transversal. Se utilizó los cuestionarios IPAQ y WHOQOL-BREF. Resultados: se observó que en la mayoría de los participantes se encontraban en nivel vigoroso de actividad física. Se halló que la mayoría trabaja de manera sedentaria entre menor o igual de 4 al día con una mediana de edad de los participantes de 37 años. Respecto a la calidad de vida en el domino de salud física y psicológica "bueno", relaciones sociales "regular" y medio ambiente "malo". Conclusiones: en nuestro estudio no se encontró una asociación significativa entre el nivel de actividad física y la percepción de la calidad de vida en el personal administrativo durante la pandemia del COVID-19.

PALABRAS CLAVE: Actividad física, calidad de vida, personal administrativo, COVID-19.

\section{SUMMARY}

Physical activity and the perception of quality of life are important to understand the problems of sedentary lifestyle, non-communicable diseases and physical inactivity in the population. COVID-19, the cause of the health emergency according to WHO. In Peru, the established state of health emergency forced them to face work challenges, therefore, teleworking began as a work alternative, which is associated with a sedentary life without physical activity and chronic pain with the biopsychosocial aspect. Objective: To determine the association between the level of physical activity and the perception of quality of life in administrative staff during the COVID-19 pandemic at a Private University of Peru. Material and methods: an observational, analytical cross-sectional study. The IPAQ and WHOQOL-BREF questionnaires. Results: The majority of the participants are at the vigorous level of physical activity. It was found that the majority work sedentary between less than or equal to 4 a day with a median age of the

Escuela de Tecnología Médica, Facultad de Medicina, Universidad Peruana Cayetano Heredia. Lima, Perú

2 Universidad Nacional de Educación Enrique Guzmán y Valle. Lima, Perú 
participants of 37 years old. Regarding the quality of life in the domain of health physical and psychological health, social relationships are "regular" and environment is "bad". Conclusions: In our study, no significant association was found between the level of physical activity and the perception of quality of life in administrative personnel during the COVID-19 pandemic.

KEYWORDS: Physical activity, quality of life, administrative staff, COVID-19.

\section{INTRODUCCIÓN}

A nivel mundial, evaluar la actividad física en las personas y su calidad de vida, han sido muy importante para conocer los problemas que genera el sedentarismo y la inactividad física en la salud de las poblaciones. Ante el ascenso de las enfermedades crónicas no transmisibles, la Organización Mundial de la Salud (OMS) diseñó dos cuestionarios con el fin de evaluar la actividad física y calidad de vida como medida de prevención en el marco de la salud $(1,2)$. En la escala mundial, el $23 \%$ de los adultos no se mantienen suficientemente activos (3).

La OMS define como actividad física al movimiento que genera el cuerpo y que es producido por el sistema musculoesquelético precisando gasto energético. Según la Organización Panamericana de la Salud demuestran que la inactividad física puede llegar hasta el $70 \%$ de la población y esto dependerá del tipo organización de cada país (4). Se debe tener en cuenta que la actividad física tiene un significado diferente al ejercicio físico, ya que, este último es más estructurado con el fin de mejorar habilidades y destrezas, además, está inmerso dentro del ámbito de la actividad física como una subcategoría (2). Así mismo, es de conocimiento que la definición de calidad de vida es la percepción de sí mismo, en los aspectos de sus valores, metas y preocupaciones, ya que, puede afectar a los factores contextuales $(5,6)$. La valoración de la calidad de vida es analizar los resultados para resaltar los beneficios y problemas que se generan. Así mismo, ser indicadores para el uso preventivo de enfermedades crónicas no transmisibles en el sector de la salud para el control de su eficacia $(7,8)$.

El siglo XXI se enfrenta a su primera pandemia mundial después de la gripe española del siglo XX (9). El COVID-19 es un patógeno infeccioso, cuya rápida propagación es la causante de la emergencia sanitaria a nivel mundial, según el Comité de Emergencias de la OMS $(10,11)$. El virus se propagó llegando a alcanzar 188 países (12), debido a ello muchas naciones optaron por implementar medidas de vigilancia sanitarias con la finalidad de salvaguardar la salud pública de su población, así como medidas basadas en el aislamiento social, distanciamiento social entre otras. Estas medidas también fueron implementadas en Perú (10).

En el Perú, debido a la pandemia y al estado de emergencia sanitaria establecido, obligó a la población a enfrentar desafíos, no solo sanitarios sino también laborales (13), por lo cual la normatividad del teletrabajo se generó como una alternativa laboral, la cual se asocia a una vida sedentaria sin actividad física y dolores crónicos $(14,15)$.

En el transcurso de la pandemia, la población que realiza teletrabajo desde sus hogares evidencia debilidades osteomusculares debido al aumento de la inactividad física y por ende percepciones no positivas de su calidad de vida, afectando el aspecto biopsicosocial.

En las instituciones de educación superior el personal docente y administrativo se encuentran realizando teletrabajo y se ha encontrado evidencia que ésta última lleva una vida sedentaria (16), sin embargo, hay un subgrupo de personal administrativo que no realiza actividades físicas, por lo que, con la nueva normalidad y por los cambios generados, podrían ser afectados con respecto a su calidad de vida $(17,18)$.

Según el Instituto Nacional de Salud, aproximadamente el $40 \%$ de la población nacional mayor de 20 años usualmente trabaja sentado, además que el $70 \%$ de la población no realiza actividades físicas (19).

En la actualidad a nivel mundial, los estudios que relacionan la actividad física y su calidad de vida han demostrado su complementación en la población, dando beneficios en la salud. Antes de la pandemia del COVID-19 en el Perú existen estudios que relacionan la actividad física y su calidad de vida en escolares, universitarios y profesores (20), sin embargo, durante la pandemia las investigaciones basadas en la actividad física y percepción de calidad de vida cuya población 
específicamente sea el personal administrativo de cualquier institución de trabajo es escasa.

Abordar esta problemática en este contexto de pandemia del COVID-19 es de interés ya que podría ser un factor determinante para la percepción de la calidad de vida en las personas (21).

A partir de estos resultados en el presente estudio, servirán para explorar más esta relación, y pueda ser considerada como línea base para nuevas investigaciones que demuestren el valor de la actividad física en las personas respecto a su calidad de vida. Por otro lado, establecer dentro del contexto laboral, ya sea en instituciones privadas o públicas actividades que permitan mejorar la calidad de vida a nivel poblacional, especialmente en el personal que tenga labores que impliquen sedentarismo. ¿Cuál es la asociación entre el nivel de actividad y la percepción de la calidad de vida en el personal administrativo durante la pandemia del COVID-19 en una ¿Universidad Privada del Perú? El objetivo del presente trabajo fue determinar la asociación entre el nivel de actividad física y la percepción de la calidad de vida en el personal administrativo durante la pandemia del COVID-19 en una Universidad Privada del Perú.

\section{MATERIAL Y MÉTODOS}

Es un estudio observacional, con diseño analítico de corte transversal (22). La población o universo está conformada por todo el personal administrativo, que realizan trabajo remoto durante el estado de emergencia sanitaria por COVID-19 en la Universidad Peruana Cayetano Heredia, Lima.

La unidad muestral son trabajadores administrativos de la Universidad Peruana Cayetano Heredia.
El tamaño de la muestra es tipo censal, porque las unidades en la investigación fueron tomadas como muestra, por lo que se trató de utilizar el $100 \%$ de la población, que fue enviado a un aproximado de 300 personas, sin embargo, solo un tercio de la población respondieron, dándonos un tamaño de muestra de 97 sujetos que trabajan como personal administrativo en la Universidad Peruana Cayetano Heredia

\section{RESULTADOS}

El $76,29 \%$ de los encuestados eran de sexo femenino, con una mediana de edad de 37 años y rango intercuantil de 29-46. El 47,42\% del total de tiempo de trabajo remoto era menor o igual de 4 horas al día y $45,36 \%$ tenía mayor o igual a 8 horas al día (tabla 1 ). Según el cuestionario IPAQ, 43,30\% realiza un nivel de actividad física vigorosa; 33 de los encuestados con un porcentaje del 34,02\% realiza un nivel de actividad física baja. Finalmente, 22,68\% realiza un nivel de actividad física moderada (tabla 2).

Según el cuestionario WHOQOL-BREF, En los dominios salud física y psicológica ambos se encuentran en un nivel bueno, uno con $43,30 \%$ y el otro con $41,24 \%$ respectivamente; en el dominio de relaciones sociales se encontraron en un nivel "regular" con un porcentaje $46,39 \%$; en el dominio ambiente se encontró en un nivel "malo" con un porcentaje de $60,82 \%$ (tabla 3 ).

Se encontró que no existe asociación entre el nivel de actividad física y la percepción de calidad de vida del personal administrativo durante la pandemia del COVID-19, los datos de esta tabla fueron recolectados el 4 de enero y duró tres semanas. Se pudo observar que, en el dominio de salud física con relación al nivel de actividad física, el mayor porcentaje obtuvo un

Tabla 1. Características de las personas que participaron en la encuesta

\begin{tabular}{llcc}
\hline & & $\mathbf{n}$ & $\mathbf{\%}$ \\
\hline Sexo & Femenino & 22 & 55 \\
& Masculino & 18 & 45 \\
Tiempo de trabajo remoto & & & \\
& & 46 & 47,42 \\
& Menor/igual de 4 horas al día & 44 & 45,36 \\
& Mayor de 4 o menor / igual de 8 horas al día & 7 & 7,22 \\
& Mayor de 9 o menor/igual a 12 horas al día & 0 & 0 \\
\hline
\end{tabular}

Edad: $39,4 * / 12,33 * *$

Mean* / Desv. Est** 
Tabla 2. Actividad física en el personal administrativo durante la pandemia COVID 19 en una universidad privada

\begin{tabular}{llcc}
\hline & & $\mathbf{n}$ & $\mathbf{\%}$ \\
\hline \multirow{3}{*}{ Nivel de actividad física } & Bajo & 33 & 34,02 \\
& Moderado & 22 & 22,68 \\
& Vigoroso & 42 & 43,30 \\
Total & & 97 & 100 \\
\hline
\end{tabular}

Tabla 3. Calidad de vida en el personal administrativo durante la pandemia del COVID19 en una universidad privada del Perú.

\begin{tabular}{|c|c|c|c|c|}
\hline Dominios & & & $\mathrm{n}$ & $\%$ \\
\hline & \multicolumn{4}{|l|}{ Salud Física } \\
\hline & & Muy malo & 0 & 0 \\
\hline & & Malo & 5 & 5,15 \\
\hline & & Regular & 37 & 38,14 \\
\hline & & Bueno & 42 & 43,3 \\
\hline & & Muy bueno & 13 & 13,4 \\
\hline & \multicolumn{4}{|l|}{ Psicológico } \\
\hline & & Muy malo & 27 & 27,84 \\
\hline & & Malo & 2 & 2,06 \\
\hline & & Regular & 13 & 13,4 \\
\hline & & Bueno & 40 & 41,24 \\
\hline & & Muy bueno & 15 & 15,46 \\
\hline & \multicolumn{4}{|l|}{ Relaciones sociales } \\
\hline & & Muy malo & 2 & 2,06 \\
\hline & & Malo & 10 & 10,31 \\
\hline & & Regular & 45 & 46,39 \\
\hline & & Bueno & 31 & 31,96 \\
\hline & & Muy bueno & 9 & $9 ., 28$ \\
\hline & \multicolumn{4}{|l|}{ Ambiente } \\
\hline & & Muy malo & 5 & 5,15 \\
\hline & & Malo & 59 & 60,82 \\
\hline & & Regular & 32 & 32,99 \\
\hline & & Bueno & 1 & 1,03 \\
\hline & & Muy bueno & 0 & 0 \\
\hline
\end{tabular}

resultado "bueno" y "vigoroso" respectivamente con un porcentaje de $47,62 \%$, ya que $\mathrm{p}=0,308$ (tabla 4 ).

En el dominio psicológico y su relación con el nivel de actividad física, el mayor porcentaje obtuvo un resultado "bueno" y "moderado" respectivamente, con un porcentaje de $59,09 \%$, ya que $p=0,225$.
En el dominio de relaciones sociales en relación al nivel de actividad física el mayor porcentaje obtuvo un resultado "regular" y "moderado" respectivamente con un porcentaje de $50 \%$, ya que $\mathrm{p}=0,112$.

En el dominio ambiente en relación al nivel de actividad física el mayor porcentaje obtuvo un 
Tabla 4. Asociación entre el nivel de actividad física y la percepción de la calidad de vida.

\begin{tabular}{|c|c|c|c|c|c|c|c|c|}
\hline & \multicolumn{7}{|c|}{ Nivel de actividad física } & \multirow[t]{2}{*}{$\mathbf{p}^{*}$} \\
\hline & & $\begin{array}{c}\text { Bajo } \\
\text { n }\end{array}$ & $\begin{array}{c}\text { Moderado } \\
\%\end{array}$ & $\begin{array}{c}\text { Vigoroso } \\
\text { n }\end{array}$ & $\%$ & $\mathbf{n}$ & $\%$ & \\
\hline \multirow[t]{6}{*}{ Salud Física } & & & & & & & & 0,308 \\
\hline & Muy malo & 0 & 0 & 0 & & 0 & & \\
\hline & Malo & 2 & 6,06 & 0 & 0 & 3 & 7,14 & \\
\hline & Regular & 15 & 45,45 & 11 & 50 & 11 & 26,19 & \\
\hline & Bueno & 14 & 42,42 & 8 & 36,36 & 20 & 47,62 & \\
\hline & Muy bueno & 2 & 6,06 & 3 & 13,64 & 8 & 19,05 & \\
\hline \multirow[t]{6}{*}{ Psicológico } & & & & & & & & 0,225 \\
\hline & Muy malo & 12 & 36,36 & 5 & 22,73 & 10 & 23,81 & \\
\hline & Malo & 0 & 0 & 0 & 0 & 2 & 4,76 & \\
\hline & Regular & 7 & 21,21 & 2 & 9,09 & 4 & 9,52 & \\
\hline & Bueno & 8 & 24,24 & 13 & 59,09 & 19 & 45,24 & \\
\hline & Muy bueno & 6 & 18,18 & 2 & 9,09 & 7 & 42 & \\
\hline \multirow[t]{6}{*}{ Relaciones sociales } & & & & & & & & 0,112 \\
\hline & Muy malo & 0 & 0 & 0 & 0 & 2 & 0 & \\
\hline & Malo & 6 & 18,18 & 3 & 13,64 & 1 & 2,38 & \\
\hline & Regular & 16 & 48,48 & 11 & 50 & 18 & 42,86 & \\
\hline & Bueno & 9 & 27,27 & 8 & 36,36 & 14 & 33,33 & \\
\hline & Muy bueno & 2 & 6,06 & 0 & 0 & 7 & 16,67 & \\
\hline \multirow[t]{6}{*}{ Ambiente } & & & & & & & & 0,772 \\
\hline & Muy malo & 3 & 9,09 & 0 & 0 & 2 & 4,76 & \\
\hline & Malo & 21 & 63,64 & 14 & 63,64 & 24 & 57,14 & \\
\hline & Regular & 9 & 27,27 & 8 & 36,36 & 15 & 35,71 & \\
\hline & Bueno & 0 & 0 & 0 & 0 & 1 & 2,38 & \\
\hline & Muy bueno & 0 & 0 & 0 & 0 & 0 & 0 & \\
\hline
\end{tabular}

Valores: * Prueba de Fisher

resultado "malo", sin embargo, fueron similares en "bajo" y "moderado" con un porcentaje de $63,64 \%$, ya que $\mathrm{p}=0,772$.

En comparación entre el nivel de actividad física y las características sociodemográficas del personal administrativo se observa una diferencia entre el género femenino y masculino, ya que el porcentaje del nivel vigoroso de los varones es mayor por $17,3 \%$, a diferencia del nivel bajo ya que las mujeres representan un porcentaje mayor, por ende, el sexo femenino realiza menor actividad física., pero no es significativo "p" valor $=0,148$ (tabla 5).

En el tiempo de trabajo remoto el resultado con mayor porcentaje es $47,83 \%$ siendo mayor a 4 horas o menor o igual de 8 horas al día, estas son las personas realizan una actividad vigorosa. Los encuestados que se encuentran en nivel moderado de actividad física trabajan igual o menor a 4 horas al día. Finalmente, las personas que presentan un nivel bajo de actividad física trabajan mayor de 9 o mayor o igual a 12 horas al día, pero no es significativo "p" valor $=0,722$.

Los encuestados con relación a la edad presentan un nivel moderado de actividad física.

En comparación entre calidad de vida y las características sociodemográficas del personal administrativo se observa en el dominio de salud física que el género femenino presenta un nivel regular y bueno a diferencia del género masculino que se encuentran en un nivel bueno, pero no es significativo "p" valor $=0,115$ (tabla 6$)$. 
Tabla 5. Comparación entre actividad física y las características sociodemográficas.

\begin{tabular}{|c|c|c|c|c|c|c|c|c|}
\hline & & \multicolumn{6}{|c|}{ Nivel de Actividad Física } & \multirow[t]{3}{*}{$\mathbf{p}^{*}$} \\
\hline & & \multicolumn{2}{|c|}{ Bajo } & \multicolumn{2}{|c|}{ Moderado } & \multicolumn{2}{|c|}{ Vigoroso } & \\
\hline & & $\mathbf{N}^{\circ}$ & $\%$ & $\mathbf{N}^{\circ}$ & $\%$ & $\mathbf{N}^{\circ}$ & $\%$ & \\
\hline \multirow[t]{4}{*}{ Sexo } & & & & & & & & 0,148 \\
\hline & Femenino & 29 & 39,19 & 16 & 21,2 & 29 & 39,19 & \\
\hline & Masculino & 4 & 17,39 & 6 & 26,09 & 13 & 56,52 & \\
\hline & & & & & & & & 0,722 \\
\hline \multirow{3}{*}{$\begin{array}{l}\text { Tiempo } \\
\text { de trabajo } \\
\text { remoto }\end{array}$} & $\leq 4$ horas & 2 & 28,57 & 3 & 42,86 & 2 & 28,57 & \\
\hline & $\begin{array}{l}>4 \text { o } \leq 8 \\
\text { horas }\end{array}$ & 15 & 32,61 & 9 & 19,57 & 22 & 47,83 & \\
\hline & $\begin{array}{l}->9 \text { o } \leq 12 \\
\text { horas }\end{array}$ & 16 & 36,36 & 10 & 22,73 & 18 & 40,91 & \\
\hline Edad & & $39 * *$ & $31-48 * * *$ & $40,5^{* *}$ & $31-46 * * *$ & $35^{* *}$ & $28-47 * * *$ & $0,50 * * * *$ \\
\hline
\end{tabular}

En el dominio psicológico en relación con el género femenino presentan mayor porcentaje en el nivel bueno a diferencia del género masculino, pero no es significativo "p" valor $=0,72$.

En el dominio relaciones sociales en relación con el género femenino presenta mayor porcentaje en el nivel regular a diferencia del género masculino, pero no es significativo "p" valor $=0,561$.

En el dominio ambiente en relación con el género ambos presentan un nivel malo siendo este su porcentaje mayor, pero no es significativo " $\mathrm{p}$ " valor $=$ 0,398 .

En el tiempo de trabajo remoto en relación con el dominio de salud física el mayor porcentaje es de las personas que trabajan mayor de 9 o menor o igual a 12 horas al día, obteniendo un nivel bueno, pero no es significativo "p" valor $=0,56$.

En el dominio psicológico hay un mayor nivel en los encuestados que trabajan mayor de nueve horas al día, o menor o igual de 12 horas "bueno", pero no es significativo "p" valor $=0,41$.

En el dominio de relaciones sociales hay un mayor nivel en los encuestados que trabajan mayor de nueve horas al día, o menor o igual de 12 horas es "regular", pero no es significativo "p" valor $=0,44$.

En el dominio ambiente hay un mayor nivel en los encuestados que trabajan mayor de nueve horas al día, o menor o igual de 12 horas "malo", pero no es significativo " $\mathrm{p}$ " valor $=0,92$.

En relación a la edad, con la calidad de vida en el dominio de salud física presenta una mediana de 48 años con rango intercuantil de 40-52 y se encuentran en el nivel "malo".

En el dominio psicológico presenta una mediana de 45,5 con rango intercuantil de $32-59$ y se encuentran en el nivel "malo".

En el dominio relaciones sociales presenta una mediana de 48,5 años con rango intercuantil de 49-53 y se encuentran en el nivel "malo".

En el dominio de ambiente presenta una mediana de 55 años con rango intercuantil de 40-64 y se encuentran en el nivel "muy malo".

\section{DISCUSIÓN}

Los datos obtenidos de la presente investigación mediante la aplicación de los instrumentos de IPAQ y WHOQOL- BREF en una muestra de 97 encuestados pertenecientes al área administrativa de la Universidad Peruana Cayetano Heredia.

Los resultados hallados muestran que la práctica de actividad física realizada por el personal administrativo no se asocia significativamente con la percepción de la calidad de vida. El valor "p" revela que no hay asociación entre las variables del estudio, 
mostrando un coeficiente por dominios según el cuestionario de calidad de vida, en la cual la Salud física es $\mathrm{p}=0,308$; Psicológico $\mathrm{p}=0,225$; Relaciones sociales $\mathrm{p}=0,112$ y Ambiente $\mathrm{p}=0,772$; por lo que la muestra de significancia del valor $p$ sale mayor a 0,05 , donde indica que no existe una asociación.

Estos datos muestran que realizar actividad física no se asocia con la calidad de vida en el personal administrativo, toda vez que la mayoría presenta una calidad de vida entre buena y regular, sin embargo, presentan un nivel de actividad física vigoroso. Así mismo, según un estudio que se realizó en Ecuador por Luis Quishpe, manifiesta que, sí existe una asociación, este estudio fue hecho con una población de 151 personas, a diferencia de nuestra investigación, debido a las limitaciones llegamos a 97 encuestados, sin embargo, este estudio sigue la misma prevalencia al nuestro, ya que hay mayor cantidad de mujeres que hombres, pero a diferencia de nuestros resultados, finalmente el autor del estudio encontró que la mayoría no realizaba actividad física y se encuentran en un nivel bajo debido a su mala calidad de vida (25).

En el caso de la actividad física del personal administrativo se encuentran en un nivel vigoroso con un porcentaje de' $43.30 \%$, sin embargo, muy cerca con $34.02 \%$ se encontraban en bajo, por consiguiente, se puede intuir que las personas que se encontraban en un nivel moderado en un mediano plazo podrían variar su actividad física. Según Jaime Nieto, en un estudio que realizó en Bogotá, la mayoría de sus participantes se encontraban en un nivel vigoroso con $53.8 \%$, seguido de un nivel moderado $42.3 \%$, sin embargo, en el nivel bajo presentaban un $0.76 \%$ por lo que menciona que ese porcentaje no camina durante 10 minutos durante el día (26).

Finalmente, en relación a la calidad de vida podemos demostrar que en los dominios de Salud física y Psicológico se encuentran en un nivel bueno, sin embargo, en relaciones sociales y ambiente son regulares y malos respectivamente, estos bajos resultados se pueden relacionar con la falta de interacción con amigos y parejas de manera presencial, ya que las normas en Perú no permitían reuniones sociales, igualmente en el caso del medio ambiente se puede relacionar con la inseguridad, falta de trabajos, días con inmovilización social y el pésimo servicio sanitario, ya que estos incrementaba al haber mayor contagio del COVID-19, por otro lado, al comparar los géneros, el masculino presentaba un mayor nivel a diferencia al femenino, respecto al tiempo de trabajo remoto el estudio demuestra que a mayor tiempo de trabajo su calidad mejoraba en algunos dominios. Un estudio en México demostró que los niveles de calidad de vida se encontraban bajos, agregando que a mayor tiempo de trabajo presentaban un nivel menor a lo esperado, mientras que en la comparación al sexo la mayor cantidad lo relacionaron al género femenino ya que hubo una mayor frecuencia de respuesta al cuestionario (27).

Finalmente, en Chile, Yeni Concha Cisternas encontró que los estudiantes universitarios activos mantienen un moderado/alto nivel de AF presentando una mejor $\mathrm{CV}$ global y en salud, y en los dominios físico, psicológico, medio ambiente al compararlos con estudiantes inactivos (21).

\section{CONCLUSIÓN}

En nuestro estudio no se encontró una asociación significativa entre el nivel de actividad física y la percepción de la calidad de vida en el personal administrativo durante la pandemia del COVID-19 en una Universidad Privada del Perú.

El personal administrativo realiza actividad física vigorosa durante la pandemia del COVID-19. La percepción de la calidad de vida en el personal administrativo durante la pandemia del COVID-19 fue buena para los dominios salud y psicológica, regular para el dominio relaciones sociales y malo para el dominio ambiente. En nuestro estudio no se encontró asociación significativa entre el nivel de actividad física según las características sociodemográficas del personal administrativo durante la pandemia del COVID-19.

No encontramos asociación entre las características sociodemográficas del personal administrativo.

\section{Correspondencia}

Vilma Violeta Lizana Villegas

Correo electrónico: vilma.lizana.v@upch.pe

Declaración de conflicto de interés: Los autores del proyecto de investigación declaramos no tener ningún tipo de conflicto de intereses, ni ninguna relación económica, personal, política, interés financiero ni académico que pueda influir en nuestro juicio. También, afirmamos no haber recibido ningún tipo de beneficio monetario, bienes ni subsidios de alguna fuente que pudiera tener interés en los resultados de esta investigación. 


\section{REFERENCIAS BIBLIOGRÁFICAS}

1. Mantilla S, Gómez-Conesa A. El Cuestionario Internacional de Actividad Física. Un instrumento adecuado en el seguimiento de la actividad física poblacional. Rev. Iberoamericana Fisioterapia y Kinesiología. 2007;10(1):48-52.

2. Torres M, Quezada M, Rioseco R, Ducci ME. Calidad de vida de adultos mayores pobres de viviendas básicas. Estudio comparativo mediante el uso de Whoqol-Bref. Rev Med Chile 2008; 136:325-333.

3. Organización Mundial de la Salud. 10 datos Sobre La Actividad Física. Ginebra: Organización Mundial de la Salud; 2017. (Citado el 13 de setiembre del 2020) Disponible en: https://www.who.int/features/ factfiles/physical_activity/es/

4. Organización Panamericana de la Salud. Plan de acción mundial sobre actividad física 2018-2030. Más personas activas para un mundo sano. Washington D.C: Organización Panamericana de la Salud; 2019. (Citado el 13 de setiembre del 2020). Disponible en: https://iris.paho.org/bitstream/hand le/10665.2/50904/9789275320600_spa.pdf

5. Fleck MP, Louzada S, Xavier M. Application of the Portuguese version of the abbreviated instrument of quality life Whoqol-bref. Rev Saude Publica. 2000;34(2):178-183.

6. Ardila R. Calidad de vida: una definición integradora. Revista Latinoamericana de Psicología. 2003; 35(2): 161-164. (Citado el 13 de setiembre del 2020). Disponible en: https://www.redalyc.org/articulo. oa? $\mathrm{id}=80535203$

7. Barboza E. Prevalencia de factores de riesgo para enfermedades crónicas no transmisibles en Perú. Revista Cuidarte. 2020; 11(2): 1-11.

8. Ramírez-Vélez R. Actividad física y calidad de vida relacionada con la salud: revisión sistemática de la evidencia actual. Revista Andaluza de Medicina del Deporte. 2010; 3(3):110-120. (Citado el 13 de setiembre del 2020). Disponible en: https://www. redalyc.org/articulo.oa?id $=323327663006$

9. Rivero-Dolores MD, Carbonetti A. La "gripe española" en perspectiva médica: los brotes de 19181919 en la escena científica argentina. Revista Ciencia Salud. 2016;14(2):281-93.

10. Organización Mundial de Salud. Preguntas y respuestas sobre la enfermedad por coronavirus (COVID-19); Ginebra: Organización Mundial de Salud; 2020 (Citado el 13 de setiembre del 2020). Disponible en: https://www.who.int/es/emergencies/ diseases/novel-coronavirus-2019/advice-for-public/ q-a-coronaviruses

11. Wang C, Horby P, Hayden F, Gao G. A novel coronavirus outbreak of global health concern. Lancet. 2020; 395 (10223): 470-473.

12. Munayco C, Chowell G, Tariq A, Undurraga E,
Mizumoto K. Riesgo de muerte por edad y sexo por COVID-19 en Perú, marzo-mayo de 2020. Envejecimiento (Albany NY). 2020; 12(14):1386913881.

13. Sanchez I. Medidas legales adoptadas en el ámbito laboral peruano debido al COVID-19. Cielo Laboral. 2020; 1: 1-4. (Citado el 13 de setiembre del 2020). Disponible en: http://www.cielolaboral.com wp-content/uploads/2020/04/peru_noticias_cielo_ coronavirus.pdf

14. Zheng C, Huang WY, Sheridan S, Sit CH, Chen XK, Wong SH. COVID-19 Pandemic Brings a Sedentary Lifestyle in Young Adults: A Cross-Sectional and Longitudinal Study. Int J Environ Res Public Health. 2020;17(17): E6035.

15. García-Salirrosas E, Sánchez-Poma R. Prevalencia de trastornos musculoesqueléticos en docentes universitarios que realizan teletrabajo en tiempos de COVID-19. An Fac med.2020;81(3). (Citado el 13 de setiembre del 2020). Disponible en: https:// revistasinvestigacion.unmsm.edu.pe/index.php/ anales/article/view/18841

16. Headley S, Hutchinson J, Wooley S, Dempsey K, Phan K, Spicer G. Subjective and objective assessment of sedentary behavior among college employees. BMC. 2018; 18:768.

17. Shanthanna H, Strand NH, Provenzano DA, et al. Caring for patients with pain during the COVID-19 pandemie: consensus recommendations from an international expert panel. Anaesthesia.2020;75(7): 935-944.

18. Lesser IA, Nienhuis CP. The Impact of COVID-19 on Physical Activity Behavior and Well-Being of Canadians. Int J Environ Res Public Health. 2020; 17(11): 3899.

19. Morales, Añez RJ, Suarez CA. Nivel de actividad física en adolescentes de un distrito de la región Callao. Rev Peru Med Exp Salud Publica. 2016;33(3):471-7.

20. Taylor P, Pediši Ž, Rakovac M, Titze S, Juraki D, Oja P. European Journal of Sport Science Domainspecific physical activity and health-related quality of life in university students. Eur J Sport Sci. 2014;14(5): 492-9.

21. Concha-Cisternas Y, Castillo-Retamal M, GuzmánMuñoz E. Comparación de la calidad de vida en estudiantes universitarios según nivel de actividad física. Univ. Salud. 2020; 22(1):33-40

22. Parreño A. Metodología de Investigación en salud. Riobamba: Escuela Superior Politécnica de Chimborazo; 2016. (Citado el 13 de setiembre del 2020). Disponible en: http://cimogsys.espoch. edu.ec/direccion-publicaciones/public/docs/ books/2019-09-17-224845-metodolog\% C3\%ADa\%20de\%201a\%20investigaci\%C3\% B3n\%20en\%20salud-comprimido.pdf

23. Craig C, Marshall A, Sjostrom M, et al. International 
physical activity questionnaire: 12-country reliability and validity. Med Sci Sports Exerc. 2003; 35(8): 1381-95. doi: 10.1249/01.MSS.0000078924.61453. FB

24. Espinoza I, Osorio P, Torrejón MJ, Lucas-Carrasco R, Bunout D. Validación del cuestionario de calidad de vida (WHOQOL-BREF) en adultos mayores chilenos. Rev Med Chile. 2011; 139: 579-586.

25. Quishpe L, Zamora J. Calidad de vida y niveles de actividad física en el personal administrativo de la universidad nacional de Chimborazo. Tesis de Pregrado. Riobamba, Ecuador: Universidad Nacional de Chimborazo; 2017.
26. Nieto JL, Angulo JJ, Jarava D. Niveles de actividad física en el personal administrativo del área de desarrollo e investigación de la sede principal Uniminuto. Tesis de Licenciatura. Bogota, Colombia: Corporación Universitaria Minuto de Dios; 2017.

27. Lulo K, Pardo R. Percepción de la Calidad de Vida en Médicos Anestesiólogos de Instituciones Públicas de Salud en Toluca, Méx. Tesis de Postgrado. Ciudad de Mexico: Universidad Autónoma del Estado de México; 2014.

Recibido: $10 / 12 / 2020$ Aceptado: $13 / 03 / 2021$ 